\title{
Diferencias en las funciones ejecutivas de estudiantes de bachillerato con distinto grado de marginación social
}

\author{
Ejecutive functions differences in bachelor's students with different level of \\ social margination
}

\author{
Sandybell González Lugo \\ José Concepción Gaxiola Romero \\ Cynthia Lorenia Aranda Corrales \\ Édgar René Valenzuela Hernández \\ Universidad de Sonora
}

\section{Resumen}

Las funciones ejecutivas son habilidades cognitivas que permiten la regulación y planificación de la conducta. En relación a los niños, se ha documentado que éstos pueden verse afectados por las carencias del contexto social. El objetivo del estudio es determinar si existen diferencias estadísticamente significativas en las funciones ejecutivas de adolescentes con distinto grado de marginación social. Se realizó un estudio transversal con 120 estudiantes de bachillerato pertenecientes a un sector de marginación media-alta y 120 pertenecientes a un sector de baja marginación. Se evaluaron las características sociodemográficas y la percepción de los riesgos contextuales de vecindario y escuela. Finalmente, se evaluaron las funciones ejecutivas con base en el Test de Clasificación de Cartas de Wisconsin, la torre de Londres, tarea tipo Go-No Go, prueba de memoria de la escala Weschler y un test de fluidez verbal. Los datos se analizaron en el paquete estadístico SPSS. Se realizó estadística descriptiva para las variables sociodemográficas. Además, fue aplicada la prueba $t$ de Student para determinar si existen diferencias significativas entre los grupos. Los resultados mostraron que los adolescentes con mayor grado de marginación social, tienen un reducido ingreso mensual, menos años de escolaridad paterna/materna y escasa habilidad en las pruebas de funciones ejecutivas en comparación con los jóvenes que viven en el sector de menor marginación social, quienes presentaron mejores puntajes en las pruebas de funciones ejecutivas y menor percepción de riesgos contextuales.

Palabras clave: adolescentes, funciones ejecutivas, marginación social, fluidez verbal, memoria de trabajo.

Nota del autor

Sandybell González Lugo, Posgrado en Ciencias Químico Biológicas y de la Salud, Universidad de Sonora (UNISON); José Concepción Gaxiola Romero, Departamento de Psicología, UNISON; Cynthia Lorenia Aranda Corrales, Posgrado Integral en Ciencias Sociales, UNISON; Edgar René Valenzuela Hernández, Posgrado en Ciencias Químico Biológicas y de la Salud, UNISON.

La correspondencia en relación con este artículo debe dirigirse a Sandybell González Lugo, Posgrado en Ciencias Químico Biológicas y de la Salud, UNISON, bulevar Luis Encinas y Rosales s/n, colonia Centro, C.P. 83000, Hermosillo Sonora, México.

Dirección electrónica: sandybell.gonzalezl@gmail.com 


\begin{abstract}
Executive functions are cognitive abilities related to regulation and planning of behavior. It has been documented that executive functions in children could be affected by deficiencies in social context. The aim of this study is to determine if there are significant differences in executive functions in adolescents with different level of social marginalization. It was developed a transversal study with 240 students, 120 coming from a mid-high socioeconomic marginalization context, and 120 students living in a low marginalization context. It was evaluated students' socio-demographic characteristics and their perception of neighborhood and school as risk contexts. Finally, it were evaluated executive functions using Wisconsin Card Sorting Computer Test, London Tower, a task Go-No Go, Weschler Memory Test and a verbal fluency test. Data were analyzed with SPSS, using descriptive statistics for socio-demographic variables and $t$ Student Test was applied to determine if there were significant differences between groups. Result showed that adolescent from the group of mid-high socioeconomic marginalization reported less income per month, their mothers and fathers had less schooling and the executive functions of that group, compared to adolescents living with less marginalization, had lower punctuations in the test applied. In addition, the adolescents with less social marginalization showed better scores in executive functions and less perception of contextual risks.

Keywords: adolescents, executive functions, social marginalization, verbal fluency, work memory.
\end{abstract}

La marginación social es considerada un fenómeno multidimensional, donde el modelo de producción económica implica un estado de desigualdad y exclusión de los beneficios y oportunidades de desarrollo para ciertos grupos sociales (Consejo Nacional de Población [CONAPO], 2010). Esta inequidad se refleja tanto en ingresos monetarios como en el acceso a bienes y servicios básicos u obligatorios, por ejemplo, la educación, la vivienda y la salud. A consecuencia de las desigualdades sociales, las comunidades marginadas constituyen grupos vulnerables cuya susceptibilidad no es resultado de una decisión individual, sino de las carencias existentes en su contexto social (Stern, 2003).

Es de esperarse que los vecindarios ubicados en sectores con marginación se caractericen por la pobreza, delincuencia y desorganización, lo cual se convierte en un factor desventajoso para todos sus habitantes, sobre todo, para los adolescentes, a quienes los contextos social, ambiental y cultural, les afectan de manera decisiva en su desarrollo psicosocial (Dos Reis, Correa, Mendes, Henrique, \& Faira, 2013).

Entre las consecuencias psicológicas del mencionado contexto social negativo, se encuentran, principalmente, aspectos afectivos y conductuales como la depresión y autoestima, dentro de los primeros (Haney, 2007), y la dependencia a sustancias y problemas de conducta, en los segundos (Caughy, Leonard, Beron, \& Murdoch, 2013); sin embargo, las consecuencias cognitivas que genera el contexto social, según los riesgos percibidos a nivel de vecindario, han sido poco evaluadas. Los estudios tienden a enfocarse, por ejemplo, 
en el nivel socioeconómico individual sobre el desarrollo cognitivo (Hackman, Farah, \& Meany, 2010), no obstante, el estatus socioeconómico además del ingreso monetario, la escolaridad y ocupación de los padres, incluyen aspectos físicos y psicosociales del entorno (Evans, 2004; Hackman \& Farah, 2009). En ese sentido, la tendencia en investigación, se centra en variables que involucran la evaluación de las características del contexto físico y social. La marginación social refleja dichas características al tomar en cuenta dimensiones como vivienda, educación, ingresos monetarios, distribución de la población y sus formas de exclusión(González, Vega, Cabrera, Romero, \& Vega, 2011). Los niveles de marginación social (CONAPO, 2010), permiten evaluar las desventajas sociales entre diversos grupos y analizar las implicaciones de dichas desigualdades, sobre las características cognitivas de los adolescentes que viven en estos climas desventajosos (Hill, Burdette, Jokinen-Gordon, \& Brailsford, 2013).

Las secuelas de la marginación social están presentes desde la niñez hasta las etapas posteriores del desarrollo psicológico; las personas con desventajas sociales tienden a presentar resultados inferiores en pruebas de inteligencia y rendimiento en comparación con sus pares que no presentan dichas desventajas, inclusive se ha reportado que las disimilitudes en el desarrollo cognitivo entre estos dos grupos sociales son mayores que las diferencias reportadas en el área de la salud física (Farah et al., 2006).
Los hallazgos en investigación que relacionan el desarrollo cognitivo con el nivel socioeconómico y las características medio ambientales (indicadores de marginación social), demuestran que las funciones ejecutivas del sistema prefrontal parecen especialmente afectadas por las inequidades sociales a edad temprana(Hackman etal., 2010), pero se requiere profundizar en estudios que relacionen dichas variables durante la adolescencia. Las funciones ejecutivas incluyen un grupo de habilidades cognoscitivas cuyo objetivo principal es facilitar la adaptación del individuo a situaciones nuevas y complejas (Rosselli, Jurado, \& Matute, 2008), y presentan un importante papel en el control emocional y en la interacción social de las personas.

Diversos procesos subyacen a las funciones ejecutivas, entre éstos se encuentran la anticipación de tareas, el planteamiento de metas, la planeación, la iniciación de las actividades, la autorregulación, la flexibilidad mental y el control atencional, además, el uso de la retroalimentación, la inhibición y el mantenimiento de información o memoria de trabajo. El desarrollo de las funciones ejecutivas se relaciona con la maduración del lóbulo frontal, que es relativamente inmaduro en el recién nacido, pero continúa su maduración y desarrollo durante la niñez y hasta la adolescencia (Anderson, 2002).

La capacidad para tomar decisiones y resolver situaciones novedosas, así como el juicio ético y la creatividad se han relacionado con la corteza prefrontal, por lo cual, se afirma que 
en las funciones ejecutivas se organizan las conductas y las funciones cognitivas exclusivamente humanas (Tirapu, Muñoz, Pelegrín, \& Albéniz, 2005). Estas funciones son útiles para coordinar capacidades cognitivas, además de la regulación emocional y conductual frente a diversas demandas ambientales.

Algunos estudios señalan que niños procedentes de familias con situaciones socioeconómicas favorables, manifiestan un mejor desempeño en las funciones ejecutivas, que aquellos procedentes de familias con condición socioeconómica baja (Hook, Lawson, \& Farah, 2013). Estos hallazgos también fueron reportados por Arán (2011), quien realizó un estudio con niños de entre 7 y 12 años, donde encontró diferencias estadísticamente significativas entre grupos de medio alto y bajo nivel socioeconómico, en función de la profesión del padre $y$ en el nivel educativo de la madre.

Las funciones ejecutivas son procesos cognitivos que se requieren para la resolución de problemas, regulación del afecto, motivación y comprensión social (Zelazo, Qu, \& Müller, 2005). Se llevan a cabo a través de subprocesos como los mencionados anteriormente, además de la memoria de trabajo, el control inhibitorio, la flexibilidad cognitiva, entre otros.

A continuación se describen las funciones ejecutivas que se evaluaron en este estudio:

Categorización. Los procesos de categorización se definen como la capacidad de clasificar objetos en categorías, e implican la capacidad de elaborar y evaluar diferentes hipótesis con el objetivo de deducir una regla de clasificación correcta (Slachevsky et al., 2005).

Inhibición. Proceso mediante el cual, el foco atencional se mantiene fijo en un tipo de estímulo y el sistema de control debe prevenir la aparición de interferencias, suscitadas por la presentación intempestiva de informaciones no pertinentes (Slachevsky et al., 2005).

Flexibilidad. Proceso mediante el cual, el foco atencional debe ser desplazado de una clase de estímulo a otro y el sistema de control debe permitir alternar entre dos set cognitivos diferentes (Slachevsky et al., 2005).

Memoria de trabajo. Sistema que mantiene y manipula temporalmente la información necesaria para realizar una tarea cognitiva (Tirapu, Muñoz, \& Pelegrín, 2002).

Planificación. Capacidad para integrar, secuenciar y desarrollar pasos intermedios para lograr metas a corto, mediano o largo plazo (Tsukiura et al., 2001). La planeación o planificación es la función ejecutiva que permite organizar sus conductas de tal forma que alcancen una meta.

Fluidez verbal. Esta función se refiere a una tarea producción que requiere la puesta en marcha de los procesos subyacentes de acceso al léxico, implica también la habilidad de organización cognitiva, la capacidad de llevar a cabo una búsqueda no habitual de palabras, atención focal, atención sostenida y procesos de inhibición, entre otros. Todos estos mecanismos forman parte de las funciones ejecutivas (García et al., 2012). 
En México, existen pocas investigaciones que aborden elementos contextuales en el estudio de las funciones ejecutivas, la mayoría se enfoca en su relación con diversos trastornos como el TDAH (Flores \& Sánchez, 2012), cuestiones relacionadas con el rendimiento académico (Castillo-Parra, Gómez, \& OstroskyShejet, 2009), escolaridad, y funcionalidad social (Lázaro, Carrasco, \& Ruiz, 2011), además de centrarse mayoritariamente en población infantil (Ramos-Loyo, Taracena, SánchezLoyo, Matute, \& González-Garrido, 2011); es necesario su estudio durante etapas posteriores como la adolescencia, cuando el individuo posee mayor capacidad para interaccionar con independencia en su contexto social. Además, entre las funciones cerebrales más importantes que explican el comportamiento social de las personas, destacan las funciones ejecutivas, ya que son la estructura que subyace al comportamiento típico de los adolescentes (Martínez \& Manoiloff, 2010). De tal manera que el estudio de las funciones ejecutivas durante ese período ayudará también a comprender la conducta de los jóvenes, tal y como se ha abordado en algunos otros estudios con adolescentes mexicanos; se ha concluido que las funciones ejecutivas continúan desarrollándose en la adolescencia (Matute et al., 2008), y que en la niñez las desigualdades socioeconómicas repercuten en su desarrollo (Hook et al., 2013), pero ¿qué sucede durante la adolescencia? Más allá de las desigualdades socioeconómicas, las características del entorno que implican vivir en un sector de marginación social ¿son diferentes las funciones ejecutivas de adolescentes que viven en sectores con distinto grado de marginación social?

Dado lo anterior, el objetivo del presente estudio fue comparar algunas de las principales funciones ejecutivas (categorización, inhibición, flexibilidad, memoria de trabajo, planificación y fluidez verbal) de adolescentes con distinto grado de marginación social.

\section{Objetivos específicos}

- Evaluar la percepción de las características del contexto vecindario y escuela en adolescentes con medio-alto grado de marginación social y bajo grado de marginación social.

- Evaluar las funciones ejecutivas en adolescentes con medio-alto y bajo grado de marginación social.

- Comparar la percepción de las características del contexto vecindario y escuela y las funciones ejecutivas en adolescentes con medio-alto y bajo grado de marginación social.

\section{Hipótesis}

$\mathrm{H}_{0}$ : No existen diferencias estadísticamente significativas en las funciones ejecutivas de adolescentes del sector con medio-alto y bajo grado de marginación social.

$\mathrm{H}_{\mathrm{a}}$ : Existen diferencias estadísticamente significativas en las funciones ejecutivas de adolescentes del sector con medio-alto y bajo grado de marginación social. 


\section{Método}

\section{Participantes}

Se realizó un estudio no experimental analítico y transversal, utilizando un muestreo de tipo intencional, donde participaron voluntariamente y con previo consentimiento informado, 120 estudiantes de bachillerato, pertenecientes a un sector de riesgo, según su grado medio-alto de marginación social con base en los índices de delincuencia y criminalidad de la Dirección General de Seguridad Pública Municipal (2013), de la ciudad de Hermosillo, Sonora. La muestra anterior se comparó con 120 estudiantes de bachillerato con bajo índice de marginación social y bajos índices de delincuencia y criminalidad, según los mismos informes oficiales. El índice de marginación social calculado por CONAPO (2010), mide la intensidad global de la marginación social en un área determinada y, por lo tanto, refleja las condiciones de pobreza y exclusión de dicha área. La información para calcular dicho índice se obtiene, básicamente, de datos censales de información proveniente del Instituto Nacional de Estadística y Geografía (INEGI).

\section{Instrumentos}

Para evaluar las características socioeconómicas de los participantes se preguntó su sexo y edad, así como los años de escolaridad, edad, estado civil e ingreso mensual de los padres. Además, se agregaron escalas para evaluar las características percibidas del contexto social e instrumentos para medir las funciones ejecutivas.
Escala de percepción del contexto social: Se utilizó para corroborar que las carencias contextuales y de marginación social fueran no solamente reportadas por los indicadores sociales, sino percibidas por los adolescentes. La escala de contexto social la conforman catorce reactivos que han sido empleados en otras investigaciones con población similar en la región (Gaxiola, González, \& Contreras, 2012; Gaxiola \& González, 2012). Estos ítems evaluaron la percepción de los estudiantes acerca del contexto a nivel de vecindario y escuela, en una escala Likert de 5 puntos donde 1 corresponde a nada y 5 a demasiado. Algunos de los reactivos de esta prueba son "¿Qué tan peligrosa es tu colonia/barrio?, ¿Qué tan descuidadas están las calles y casas? y ¿Qué tanta droga se vende en tu escuela?". La confiabilidad para dicha escala oscila en valores de alfa de .78 a .80 , según las investigaciones (Gaxiola et al., 2012; Gaxiola \& González, 2012).

Test de Clasificación de Cartas Wisconsin (WCST): Se usó para evaluar las funciones ejecutivas flexibilidad e inhibición (Heaton, 1981, citado en Grodzinski \& Diamond, 1992), en su versión computarizada. La prueba ha sido estandarizada y normalizada en edades de entre los 6 y los 89 años. Este instrumento tiene un valor de alfa de 77 de acuerdo con sus autores (Heaton, 1981, citado en Grodzinski \& Diamond, 1992). El WCST mide el uso de la retroalimentación, la capacidad para modificar estrategias incorrectas, los procesos de categorización, la flexibilidad e inhibición. El test consiste en una pantalla 
con cinco estímulos donde se presentan cartas compuestas por la combinación de tres atributos: forma (triángulo, estrella, cruz y círculo), el color (rojo, azul, verde y amarillo) y la cantidad (uno, dos, tres o cuatro elementos). La tarea implica emparejar las cartas según los criterios (color, forma y número), por ejemplo, en el caso del color, dicho criterio es desconocido para el participante, quien debe descubrir el criterio a partir de la retroalimentación positiva: "correcto" o negativa: "incorrecto", la cual le es proporcionada automáticamente por el programa a través de un mensaje escrito en el monitor. Cuando el participante realiza diez respuestas correctas consecutivas consigue una categoría, y a partir de la última se cambia el criterio de clasificación sin previa advertencia. Si continúa clasificando las cartas con el criterio de la categoría anterior, va puntuando en errores perseverativos.

Las principales variables dependientes son número de categorías correctas identificadas, respuestas perseverativas (número de tarjetas que el sujeto ordena bajo una categoría anterior correcta, a pesar de la retroalimentación negativa del ordenador), errores perseverativos, cantidad total de errores y fallos de mantenimiento del set.

Torre de Londres: Esta prueba se aplicó para evaluar la capacidad de planificación. Se usó la versión computarizada de la prueba, que consiste en una tarea de planificación en la cual se presenta al participante una serie de discos de colores distintos (rojo, azul y verde), colocados en tres pilas de distinto tamaño; en una pila cabe un disco, en otra dos y en la última tres. El objetivo es que a partir de una configuración inicial el sujeto mueva los discos de tal manera que éstos queden organizados igual que en el ejemplo. Solo puede moverse un disco a la vez y el objetivo es acomodar los discos con el menor número de movimientos posibles. Entre menor es el número de movimientos realizados más eficiente es su capacidad de planificación. El valor de alfa de Cronbach de la prueba es de .83 (Shallice, 1982).

Tarea de ejecución-no ejecución (Tarea Go-No Go): Los paradigmas de ejecución-no ejecución de respuesta se han empleado para medir la inhibición, es decir, instruir a los sujetos a responder a un estímulo go (sigue), y a inhibir la respuesta ante el estímulo no go (para). En esta prueba computarizada, el participante observa en la pantalla una serie de letras $\mathrm{P}$ y $\mathrm{R}$ y debe presionar el shift derecho del teclado cuando observa la letra $\mathrm{P}$ durante los primeros 5 minutos de la prueba. En una segunda fase debe responder solamente al estímulo R, es decir, deberá inhibir la tendencia aprendida de responder ante el estímulo P. En total, se muestran 320 estímulos divididos en dos secciones de 5 minutos cada una, en ambas se muestran 128 estímulos P y 32 estímulos R. La prueba se aplica en el software $P E B L$ : The Psychology Experiment Building Language (Mueller, 2011) y obtuvo un valor de alfa de .62. 
Subtest de dígitos (directos e inversos) pertenecientes a las escalas de inteligencia de Wechsler: Esta prueba, además de explorar el span o amplitud de memoria inmediata y de trabajo, demanda concentración y la implicación de la atención ejecutiva, sobre todo, a medida que la tarea va aumentando en dificultad. La prueba de dígitos consiste en dos partes que se aplican por separado: dígitos en orden directo y dígitos en orden inverso. En los dos casos el participante escucha una serie de números y debe repetirla en el mismo orden en que se ha presentado (orden directo) o en el orden inverso. Este último caso se aplica siempre, incluso cuando el sujeto no haya puntuado en el orden directo. Se aumenta progresivamente la longitud de la serie, hasta que el participante comete dos fallos seguidos. La repetición de dígitos en forma directa e inversa es útil para evaluar la capacidad ejecutiva de la memoria operativa al evaluar la memoria de trabajo de mantenimiento en su versión directa y la memoria de trabajo manipulativa en la presentación inversa (González et al., 2008). El valor de alfa obtenido en esta prueba fue de .62 .

Fluidez verbal: Se utilizó una prueba de fluidez verbal en sus factores fluidez semántica y fonética. Dicha prueba consiste en que el participante nombre en un minuto el mayor número de elementos pertenecientes a una categoría, accediendo al sistema léxico y a la memoria semántica, al ser capaz de elegir entre diferentes opciones de producción. La categoría empleada en este trabajo fue "animales", así como las palabras que iniciaran con la letra $\mathrm{P}$, M y R, por ser las categorías más utilizadas en estudios de habla hispana. En esta prueba se contabiliza el número de palabras correctas que el individuo emite durante cada ensayo de un minuto. Hirshorn y Thompson-Schill (2006), postularon que las funciones frontales asociadas al desempeño ejecutivo en fluidez verbal son el monitoreo de la producción, la generación de estrategias de búsqueda, las funciones asociadas a la memoria de trabajo y la flexibilidad cognitiva. El valor de alfa en la prueba de fluidez verbal fue de .80 .

\section{Procedimiento}

La investigación se dividió en dos fases, en la primera se acudió a dos escuelas con distinto grado de marginación social (CONAPO, 2010), una ubicada en un sector de marginación mediaalta y otra en un sector con baja marginación social. En ambas escuelas se obtuvieron los permisos correspondientes y con previo consentimiento informado se aplicó un cuestionario a los adolescentes en el aula y horario de clases, que incluía la evaluación socio-demográfica. Este fue respondido en un tiempo aproximado de 20 minutos. La segunda fase consistió en una evaluación individual de cada uno de los participantes, esta evaluación incluía una serie de ejercicios en la computadora para evaluar las funciones ejecutivas; la duración de la evaluación completa fue de 50 minutos. Se excluyeron aquellos adolescentes que padecieran cualquier 
tipo de enfermedad y que estuvieran bajo medicación, con el objetivo de controlar que las diferencias encontradas no se debieran a alguno de estos factores. Las evaluaciones fueron grabadas en audio para más tarde verificar que todos los participantes hubieran recibido las mismas instrucciones y su ejecución no fuera afectada por algún comentario de los evaluadores. Los instrumentos fueron aplicados por un psicólogo y cuatro estudiantes de psicología, previamente entrenados para la aplicación de los instrumentos. Una vez finalizada la evaluación se agradecía a los participantes y se les solicitaba un correo electrónico a donde posteriormente les fueron enviados sus resultados.

\section{Análisis de datos}

Se escucharon las grabaciones de cada una de las evaluaciones para corroborar los datos recabados en los registros y se capturaron los resultados en el paquete estadístico SPSS versión 20.0. Se realizó la estadística descriptiva para las variables sociodemográficas. Después, se aplicó la prueba de bondad de ajuste para establecer si existían diferencias entre las características socio-demográficas de los alumnos según la escuela de procedencia, y finalmente, se aplicó la prueba $t$ de Student para evaluar las diferencias tanto de las funciones ejecutivas como de las escalas con base en el grado de marginación social de los grupos conformados.

\section{Resultados}

Los resultados indican que existen diferencias en algunos aspectos sociodemográficos entre la percepción de las características del contexto y las funciones ejecutivas de ambos grupos. En la tabla 1 se presentan las características socioeconómicas de la muestra, destacan diferencias estadísticamente significativas en lo referente al ingreso mensual, que es menor en los adolescentes que estudian en un sector de marginación media-alta, en comparación con quienes estudian en un lugar de baja marginación social.

Se encontraron también diferencias en el estado civil de los padres y en los años de escolaridad (tabla 2), elementos que implican desigualdad en nivel socioeconómico y en la estructura familiar de los grupos.

En lo referente a la percepción de las características negativas en el entorno, tanto del vecindario como de la escuela, los participantes ubicados del grupo de media-alta marginación social obtuvieron una $\bar{x}=2.43(D . S=.53)$, mientras que el grupo de baja marginación social obtuvo una $\bar{x}=2.13(D . S .=.46), t=4.457, p$ $<.0$. Esto significa que los adolescentes perciben las carencias y necesidades de sus entornos y que existen diferencias estadísticamente significativas en las características negativas percibidas del contexto, según el grado de marginación social de los estudiantes; dicha percepción es mayor en el grupo con más marginación social. 
Tabla 1

Características sociodemográficas de los adolescentes de la muestras clasificados según su grado de marginación social

\begin{tabular}{|c|c|c|c|c|}
\hline Variable & $\begin{array}{l}\text { Media-alta } \\
\text { marginación \% } \\
\mathrm{n}=115\end{array}$ & $\begin{array}{l}\text { Baja } \\
\text { marginación \% } \\
n=113\end{array}$ & $X^{2}$ & $P$ \\
\hline Sexo & & & $3.934(1)$ & .063 \\
\hline Masculino & 41.7 & 54.8 & & \\
\hline Femenino & 58.3 & 45.2 & & \\
\hline Trabaja & & & $.172(1)$ & .748 \\
\hline Sí & 22.6 & 19 & & \\
\hline No & 77.4 & 81 & & \\
\hline Vive con & & & $2.995(4)$ & .559 \\
\hline Ambos padres & 55.7 & 52.4 & & \\
\hline Madre & 25.2 & 32.5 & & \\
\hline Padre & 5.2 & 4.8 & & \\
\hline Otro & 13.9 & 7.9 & & \\
\hline Ingreso familiar+ & & & $31.898(3)$ & $000^{* *}$ \\
\hline Menos de $\$ 5,000$ & 53 & 22.2 & & \\
\hline Entre $\$ 5,000$ y $\$ 10,000$ & 32.2 & 37.3 & & \\
\hline Entre $\$ 10,000$ y $\$ 15,000$ & 6.1 & 23.8 & & \\
\hline Más de $\$ 15,000$ & 4.3 & 12.7 & & \\
\hline Estado civil madre & & & $14.235(5)$ & $.014^{*}$ \\
\hline Casada & 47.8 & 61.9 & & \\
\hline Divorciada & 16.5 & 15.9 & & \\
\hline Viuda & 3.5 & 7.1 & & \\
\hline Soltera & 10.4 & 3.5 & & \\
\hline Unión libre & 17.4 & 7.1 & & \\
\hline \multicolumn{5}{|l|}{ Estado civil del padre } \\
\hline Casado & 50.4 & 61.9 & $13.866(4)$ & $.008^{* *}$ \\
\hline Divorciado & 13.9 & 14.2 & & \\
\hline Viudo & .9 & 2.7 & & \\
\hline Soltero & 1.7 & 2.7 & & \\
\hline Unión libre & 18.3 & 3.5 & & \\
\hline
\end{tabular}

Tabla 2

Edad y escolaridad de los participantes y sus padres

\begin{tabular}{lllllll}
\hline Variable & \multicolumn{7}{l}{ Media-alta marginación } & \multicolumn{3}{l}{ Baja marginación } & & \\
& $\mathrm{n}=115$ & $\mathrm{n}=113$ & & & \\
\hline & $M$ & $D S$ & $M$ & $D S$ & $t$ & $p$ \\
Edad del adolescente & 17.32 & .74 & 7.24 & .43 & .915 & .361 \\
Años escolaridad del adolescente & 12.65 & .88 & 12.81 & .80 & -.864 & .389 \\
Edad de la madre & 41.18 & 4.82 & 44.40 & 7.54 & -3.839 & $.000^{* *}$ \\
Años escolaridad de madre & 9.95 & 2.87 & 13.89 & 4.43 & -7.982 & $.000^{* *}$ \\
Edad del padre & 44.25 & 5.99 & 46.94 & 7.57 & -2.973 & $.003^{* *}$ \\
Años escolaridad del padre & 10.44 & 3.39 & 14.80 & 3.72 & -9.228 & $.000^{* *}$ \\
\hline
\end{tabular}

$N=228$ 
En la tabla 3 se muestran los resultados de las diferentes pruebas neuropsicológicas utilizadas en la evaluación de las funciones ejecutivas para cada uno de los grupos. En todas las pruebas se encontraron diferencias estadísticamente significativas, exceptuando en la tarea tipo Go-No
Go, donde a pesar de que la media de precisión y el número de aciertos fue un poco mayor en el grupo con menor grado de marginación social, esas diferencias fueron mínimas y estadísticamente no significativas.

Tabla 3

Comparación de las funciones ejecutivas según el grado de marginación social

\begin{tabular}{|c|c|c|c|c|c|c|}
\hline \multirow[b]{2}{*}{ Prueba/variables } & \multicolumn{2}{|c|}{ Mayor marginación } & \multicolumn{3}{|c|}{ Menor marginación } & \multirow[b]{2}{*}{$p$} \\
\hline & $\mathrm{M}$ & DS & $\mathrm{M}$ & DS & $t$ & \\
\hline \multicolumn{7}{|l|}{ Test de Clasificación de Cartas } \\
\hline \multicolumn{7}{|l|}{ Wisconsin } \\
\hline Total de ensayos & 111.56 & 18.821 & 103.87 & 19.385 & 3.701 & $.000^{* *}$ \\
\hline Ensayos correctos & 68.37 & 15.672 & 72.03 & 11.850 & -2.320 & .139 \\
\hline Total errores & 36.41 & 18.897 & 28.36 & 15.293 & 4.287 & $.000^{* *}$ \\
\hline Resp. persev. & 18.40 & 9.322 & 15.05 & 11.198 & 3.522 & $.000^{* *}$ \\
\hline Errores persev. & 16.38 & 7.660 & 13.49 & 8.582 & 3.763 & $.000^{* *}$ \\
\hline Errores no pers. & 19.91 & 14.902 & 14.93 & 10.159 & 3.310 & $.003^{* *}$ \\
\hline Nivel conceptual & 54.43 & 23.531 & 64.32 & 20.435 & -4.373 & $.000^{* *}$ \\
\hline Ensayos primer Cat. & 23.35 & 31.567 & 15.52 & 10.776 & 2.838 & .926 \\
\hline Núm. categorias & 4.61 & 1.932 & 5.20 & 1.464 & -3.239 & $.001^{* *}$ \\
\hline EMS & .69 & .902 & .84 & .991 & -1.216 & .240 \\
\hline \multicolumn{7}{|l|}{ Torre de Londres } \\
\hline Número de movimientos & 84.37 & 26.959 & 71.92 & 19.426 & 3.847 & $.000^{* *}$ \\
\hline Tiempo para completar & 339.94 & 141.35 & 257.04 & 103.43 & 4.556 & $000^{* *}$ \\
\hline \multicolumn{7}{|l|}{ Tarea go/no go } \\
\hline Total aciertos & 305.03 & 9.30 & 305.25 & 12.536 & -.012 & .301 \\
\hline Total errores & 14.90 & 9.30 & 14.26 & 10.422 & .380 & .334 \\
\hline Media de precisión & .9381 & .1162 & .9493 & .082203 & -.742 & .231 \\
\hline Media de error & .0520 & .0664 & .0486 & .05925 & .300 & .383 \\
\hline \multicolumn{7}{|l|}{ Fluidez verbal } \\
\hline Total animales & 18.91 & 4.898 & 21.84 & 4.903 & -4.349 & $.000^{* *}$ \\
\hline Total palabras con $\mathrm{P}$ & 11.85 & 4.195 & 14.40 & 4.880 & -4.405 & $.000^{* *}$ \\
\hline Total palabras con $\mathrm{M}$ & 10.32 & 3.573 & 12.52 & 4.195 & -4.794 & $.000^{* *}$ \\
\hline Total palabras con $\mathrm{R}$ & 10.11 & 3.818 & 11.54 & 3.838 & -3.091 & $.001^{* *}$ \\
\hline Palabras totales & 51.20 & 13.153 & 60.309 & 13.36 & -5.424 & $.000^{* *}$ \\
\hline \multicolumn{7}{|c|}{$\begin{array}{l}\text { Test de memoria-dígitos directos } \\
\text { e inversos }\end{array}$} \\
\hline Span directo & 4.84 & .864 & 5.54 & 1.004 & -5.483 & $.000^{* *}$ \\
\hline Span inverso & 4.11 & .074 & 4.63 & 1.144 & -3.422 & $.001^{* *}$ \\
\hline
\end{tabular}

Resp. Persev = respuestas perseverativas; Errores persev. $=$ Errores perseverativos; Errores no pers. $=$ Errores no perseverativos; Nivel conceptual $=\%$ Respuestas a nivel conceptual; Ensayos primer cat. = Número de ensayos para lograr completar la primera categoría; Núm. Categorías = Número de categorías que logró completar; EMS = Errores de mantenimiento de Set.

${ }^{* *}=p<.01$. 


\section{Discusión}

Según los resultados obtenidos con el test de clasificación de cartas de Wisconsin, se observó un mayor porcentaje de errores perseverativos $(\bar{x}=16.38)$ en la escuela ubicada en el sector con mayor marginación social en comparación con la escuela de baja marginación social $(\bar{x}=$ 13.49), esto implica que los alumnos con mayor marginación social presentaron mayores dificultades para modificar estrategias en la resolución de un problema, a partir de la retroalimentación, que los adolescentes de menor marginación social. De igual forma, en lo que se refiere al porcentaje de nivel conceptual y el número de categorías completadas, se obtuvieron mayores porcentajes en los adolescentes con menor marginación social que en los de mayor, lo cual significa mayor flexibilidad cognitiva e inhibición en los adolescentes más favorecidos socioeconómicamente. Estos resultados concuerdan con otras investigaciones, donde el nivel socioeconómico tiene implicaciones importantes en el desarrollo de las funciones ejecutivas (Hackman \& Farah, 2009).

En la variable planeación medida según la torre de Londres, los adolescentes del grupo de mayor marginación social tardaron más tiempo en completar el test, a la vez que realizaron mayor número de movimientos, esto implica que el tiempo de respuesta no fue necesariamente invertido en la búsqueda de estrategias, o bien, que a pesar de buscar dichas estrategias no fueron completamente efectivas. En el grupo de menor marginación social, a pesar de tomarse menos tiempo en completar la torre, ésta fue terminada con un menor número de movimientos. Dicha variable hace referencia a la capacidad de pensar antes de actuar y, debido a su correlación con la activación del área dorso-lateral de los lóbulos frontales, es posible inferir que los adolescentes que tienen resultados inferiores en esta prueba, presentan menor desarrollo en estas áreas cerebrales (Ruocco et al., 2014).

Por otro lado, en el área de inhibición, según la tarea tipo Go-No Go, resultó que la media de precisión fue cercana a 1 y la media de error fue cercana a 0 en ambos grupos. Por lo tanto, esta prueba no identificó dificultades de inhibición en ninguno de los dos grupos. Con base en estos datos, es posible inferir que no existen problemas de impulsividad en los participantes; dichos resultados coinciden con los hallazgos encontrados por Ernst y Paulus (2005). Esto se puede explicar debido a que las tareas tipo Go-No Go evalúan la inhibición conductual a diferencia de otras pruebas que miden inhibición cognitiva, como el Priming Negativo o el control de la interferencia como la prueba Stroop (Nigg, 2000); aunque la impulsividad es una característica típica de los adolescentes, la relación entre ésta y la inhibición no ha reportado correlaciones importantes en términos conductuales, aunque sí a nivel funcional y electrofisiológico (Vázquez, 2014).

En las pruebas de fluidez verbal, ambos grupos mostraron mayor capacidad en las funciones ejecutivas cuando se evaluó la fluidez semántica, es decir, cuando se evoca un esquema cog- 
nitivo referente a una categoría específica como lo fue el caso de los animales, en comparación con la fluidez fonética, donde los esquemas cognitivos actualizan información según el sonido de las palabras y no su significado (García et al., 2012).

En todos los indicadores de la prueba de fluidez verbal, el grupo con marginación social obtuvo menor puntaje en el número de palabras mencionadas, esto significa que hay mayor capacidad en dicho grupo para poner en marcha procesos asociados al lenguaje, organización cognitiva, búsqueda y selección de elementos en sus esquemas (Hirshorn \& Thompson-Schill, 2006).

Finalmente, en lo que se refiere a memoria de trabajo, según el indicador de span inverso, los adolescentes del grupo de mayor marginación social presentaron menor capacidad para mantener la información en comparación con los adolescentes del grupo de menor marginación.

Se encontraron valores inferiores en los resultados de las funciones ejecutivas en el grupo de menor nivel socioeconómico en comparación con el grupo de mayor nivel socioeconómico, esto puede deberse a los niveles de educación de los padres, ya que el desarrollo de las funciones ejecutivas depende tanto de la maduración a través de procesos biológicos, así como de la cantidad y calidad de las experiencias de aprendizaje que proporciona el medio ambiente, por consiguiente, se ha postulado que factores socioculturales pueden influir en su desarrollo (Hackman \& Farah, 2009).
Con base en los resultados obtenidos, se sugiere que futuras investigaciones continúen evaluando las funciones ejecutivas en adolescentes y consideren la influencia del factor socioeconómico, ya que la mayoría de los estudios se han centrado en población infantil (Hook et al., 2013), con el objetivo de que, posteriormente, puedan elaborarse programas de intervención basados en evidencia empírica, cuya finalidad sea mejorar el desarrollo cognitivo de grupos socialmente vulnerables.

A partir de los hallazgos en este estudio, es recomendable promover la autorregulación, la capacidad de planeación y solución de problemas en adolescentes que viven en sectores de riesgo, dado que los indicadores de la evaluación de las funciones ejecutivas sugieren cierta desventaja en comparación con otros adolescentes que no pertenecen a sectores marginados.

Entre las limitaciones del estudio, resalta la falta de control sobre las variables biológicas, de desarrollo y hereditarias que pueden afectar las funciones ejecutivas. Se recomienda que futuros estudios incluyan un análisis biomédico para descartar daño neurológico, dislipidemias o alguna otra patología que pudiera afectar el desempeño de los adolescentes en las pruebas; además, se propone evaluar los antecedentes heredofamiliares y la historia clínica para afirmar que las diferencias se deban exclusivamente a la marginación social y no a otras causas. 


\section{Referencias}

Anderson, P. (2002). Assessment and development of executive function (EF) during childhood. Child Neuropsychology, 8(2), 71-82. doi:10.1076/chin.8.2.71.8724

Arán, F. V. (2011). Funciones ejecutivas en niños escolarizados: Efectos de la edad y del estrato socioeconómico. Avances en Psicología Latinoamericana/Bogotá (Colombia), 29(1), 98-113.

Castillo-Parra, G., Gómez, E., \& OstroskyShejet, F. (2009). Relación entre las funciones cognitivas $y$ el nivel de rendimiento académico en niños. Revista de Neuropsicología, Neuropsiquiatría y Neurociencias, 9(1), 41-54.

Caughy, M. O. B., Leonard, T., Beron, K., \& Murdoch, J. (2013). Defining neighborhood boundaries in studies of spatial dependence in child behavior problems. International Journal of Health Geographics, 12(1), 24. doi:10.1186/1476-072X-12-24

Consejo Nacional de Población [CONAPO]. (2010). Índices de marginación 2010, Colección Índices Sociodemográficos. México: CONAPO.

Dos Reis, D. C., Correa,A. T. A., Mendes, M. M., Henrique, R. A., \& Faira, M. A. M. (2013). Vulnerabilidad a la salud en la adolescencia: Condiciones socioeconómicas, redes sociales, drogas y violencia. Revista Latino Americana de Enfermagem, 21(9), 586594. Recuperado de http://www.scielo.br/ scielo.php?script $=$ sci_arttext\&pid $=\mathrm{S} 0104$ -
$11692013000200586 \& \operatorname{lng}=\mathrm{pt} \& \mathrm{t} \operatorname{lng}=\mathrm{es}$. 10.1590/S0104-11692013000200016.

Dirección General de Seguridad Pública Municipal (2013). Colonias más conflictivas según los índices de inseguridad $\mathrm{y}$ delincuencia de Hermosillo, Sonora. Reporte Electrónico, INFOMEX. Recuperado de www.infomex.org

Ernst, M., \& Paulus, M. P. (2005). Neurobiology of decision making: A selective review from a neurocognitive and clinical perspective. Biological Psychiatry, 58, 597-604.

Evans, G. W. (2004). The environment of childhood poverty. American Psychologist, 59(2), 77-92. doi: 10.1037/0003-066X.59.2.77

Farah, M. J., Shera, D. M., Savage, J. H., Betancourt, L., Giannetta, J. M., Brodsky, N. L., \& Hurt, H. (2006). Childhood poverty: Specific associations with neurocognitive development. Brain Research, 1110(1), 166174. doi: 10.1016/j.brainres.2006.06.072

Flores, H. C. L., \& Sánchez, E. L. Z. (2012). Comparación de funciones ejecutivas en muestra de niños con y sin TDAH de la ciudad de México. Revista Electrónica de Psicología Iztacala, 15(2), 663-687.

García, E., Rodríguez, C., Martín, R., Jiménez, J. E., Hernández, S., \& Díaz, A. (2012). Test de Fluidez Verbal: Datos normativos y desarrollo evolutivo en el alumnado de primaria. European Journal of Education and Psychology, 5(1), 53-64.

Gaxiola, J. C. R., \& González, L. S. (2012). Predictores del rendimiento académico y 
resiliencia en adolescentes de secundaria. En J. Palomar \& J. Gaxiola (Eds.), Estudios de Resiliencia en América Latina, 1 (pp.72-95). México: Pearson.

Gaxiola, R. J. C., González, L. S., \& Contreras, H. Z. (2012). Influencia de la resiliencia, metas y contexto social en el rendimiento académico de bachilleres. Revista Electrónica de Investigación Educativa, 14(1), 165-181.

González, M. R., González, P. I. H., Hernández, E. M., Rodríguez, S. A., Quintero, F. M., \& Morell, R. B. (2008). Evaluación neuropsicológica de la memoria en el trastorno por déficit de atención/hiperactividad: Papel de las funciones ejecutivas. Revista de Neurología, 47(5), 225-230.

González, P. G., Vega, L. M., Cabrera, P. C., Romero, V. S., \& Vega, L. A. (2011). Contexto demográfico, desigualdad social e inequidad en salud de la niñez en México. Revista Salud Pública, 13(1), 41-53.

Grodzinzki, G, M., \& Diamond, R. (1992). Frontal lobe functioning in boys with attention deficit hyperactivity disorder. Developmental Neuropsychology, 8(4), 427-445. doi: $10.1080 / 87565649209540536$

Hackman, D. A., \& Farah, M. J. (2009). Socioeconomic status and the developing brain. Trends in Cognitive Sciences, 13(2), 65-73. doi: 10.1016/j.tics.2008.11.003.

Hackman, D. A., Farah, M. J., \& Meany, M. J. (2010). Socioeconomic status and the brain: Mechanistic insights from human and animal research. Nature Reviews Neuroscience, 11, 651-659. doi: 10.1038/nrn2897

Haney, T. J. (2007). Broken Windows and self-esteem: Understandings of neighborhood poverty and disorder. Social Science, 36(3), 968994. doi: 10.1016/j.ssresearch.2006.07.003

Hill, T. D., Burdette, A. M., Jokinen-Gordon, H. M., \& Brailsford, J. M. (2013). Neighborhood Disorder, Social Support, and Self-Esteem: Evidence from a Sample of Low-income Women Living in Three Cities. City \& Community, 12, 380-395. doi: 10.1111/ cico. 12044

Hirshorn, A. E., \& Thompson-Schill, L. S. (2006). Role of the left inferior frontal gyrus in covert Word retrieval: Neural correlates of switching during verbal fluency. Neuropsychologia, 44(12), 2547-2557. doi: 10.1016/j.neuropsychologia.2006.03.035

Hook, J. C., Lawson, M. G., \& Farah, J. M. (2013). La condición socioeconómica y el desarrollo de las funciones ejecutivas. Enciclopedia sobre el desarrollo de la primera infancia, 1-8. Recuperado de http:// www.enciclopedia-infantes.com/documents/ Hook-Lawson-FarahESPxp1.pdf

Lázaro, J. C. F., Carrasco, B. T., \& Ruiz, B. C. (2011). Influencia del nivel y de la actividad escolar en las funciones ejecutivas. Interamerican Journal of Psychology, 45(2), 281-292.

Martínez, M., \& Manoiloff, L. M. V. (2010). Evaluación neuropsicológica de la función ejecutiva en adolescentes con diferentes 
patrones de consumo de alcohol. Revista Argentina de Ciencias del Comportamiento, 2(1), 14-23.

Matute, E., Chamorro, Y., Inuzentseva, O., Barrios, O., Roselli, M., \& Ardila, A. (2008). Efecto de la edad en una tarea de planificación y organización (Pirámide de México) en escolares. Revista de Neurología, 47(2), 6170.

Mueller, S. (2011). The PEBL Go/No-Go test. Computer Software. Recuperado de http:// pebl.sf.net/battery.html

Nigg, J. T. (2000). On inhibition/disinhibition in developmental psychopathology: Views from cognitive and personality psychology and a working inhibition taxonomy. Psychological Bulletin, 126(2), 220-246. doi:10.1037//0033-2909.126.2.220

Ramos-Loyo, J., Taracena, A., Sánchez-Loyo, L., Matute. E., \& González-Garrido, A. (2011). Relación entre el funcionamiento ejecutivo en pruebas neuropsicológicas y en el contexto social en niños con TDAH. Revista de Neuropsicología, Neuropsiquiatría y Neurociencias, 11(1), 1-16.

Roselli, M., Jurado, M. B., \& Matute, E. (2008). Las funciones ejecutivas a través de la vida. Revista de Neuropsicología, Neuropsiquiatría y Neurociencias, 8(1), 23-46.

Ruocco, C. A., Rodrigo, H. A., Lam, J., Di Domenico, S., Graves, B., \& Ayaz, H. (2014). A problem-solving task specialized for functional neuroimaging: Validation of the Scar- borough adaptation of the Tower of London (S-Tol) using nearinfrared spectroscopy. Frontiers in human neuroscience, 8(185), 1-13. doi:10.3389/fnhum.2014.00185

Shallice, T. (1982). Specific impairments of planning. Philosophical Transcripts of the Royal Society of London, 298(2), 199-209. doi: 10.1098/rstb.1982.0082

Slachevsky, A., Pérez, C., Silva, J., Orellana, M. L. P., Alegria, P., \& Peña, G. M. (2005). Córtex prefrontal y trastornos del comportamiento. Modelos explicativos $\mathrm{y}$ métodos de evaluación. Revista Chilena de Neuropsiquiatría, 43(2), 109-121.

Stern, D. I. (2003). Environmental Kuznets Curve. Encyclopedia of Ecological Economics (OEEE), The International Society for Ecological Economics (ISEE). Recuperado de http://isecoeco.org/pdf/stern.pdf

Tirapu, U. J., Muñoz, C. J. M., Pelegrín, V. C., \& Albéniz, F. A. (2005). Propuesta de un protocolo para la evaluación de las funciones ejecutivas. Revista de Neurología, 41(3), 177-186.

Tirapu, U. J., Muñoz, C. J. M., \& Pelegrín, V. C. (2002). Funciones ejecutivas: Necesidad de una integración conceptual. Revista de Neurología, 34(7), 673-685.

Tsukiura, T., Fujii, T., Takahashi, T., Xiao, R., Inase, M., Iijima, T., \& Okuda, J. (2001). Neuroanatomical discrimination between manipulating and maintaining processes involved in verbal working memory: A 
functional MRI study. Cognitive Brain

Research, 11, 13-21. doi: 10.1016/S09266410(00)00059-8

Vázquez, M. P. A. (2014). Efecto de la demora en la respuesta sobre la inhibición ante un contexto emocional en adolescentes con alta y baja impulsividad (Tesis de Maestría no publicada). Guadalajara, México: Universidad de Guadalajara.

Zelazo, P. D., Qu, L., \& Müller, U. (2005). Hot and cool aspects of executive function: Relations in early development. En W. Schneider, R. Schumann-Hengsteler \& B. Sodian (Eds.), Young children's cognitive development: Interrelationships among executive functioning, working memory, verbal ability, and theory of mind (pp. 7193). Nueva Jersey, USA: Lawrence Erlbaum. 